\title{
Chapter 9 \\ When Lanchester Met Richardson: The Interaction of Warfare with Psychology
}

\author{
Niall MacKay
}

\begin{abstract}
Simple dynamical systems, in the spirit of Richardson's arms race, can be used to investigate the core dynamics of various models of insurgent and multilateral war. This chapter describes two such models. The first combines Richardson's two-nation arms race with Lanchester's attrition model and Deitchman's guerrilla variant of it to create a model in which the typical long-term outcome is neither annihilation nor escalation but rather a stable fixed point, a stalemate. The scaling it implies for the force required to defeat an insurgency matches that which has been observed. The second model is of multilateral attritional war, in the spirit of Richardson's multinational arms race. We describe the case of three antagonists, whose objective is to win but, if they cannot win, to minimize their remaining opponents. In contrast to truels and triads in which the objective is survival, and the weakest actor often emerges in a position of surprising strength, here the outcome is mutual annihilation, unless one side can beat the others put together.
\end{abstract}

Much of the controversy about the value of Richardson's arms race models can be set within a wider discussion of the value of mathematical modelling generally. An excellent modern essay is Joshua Epstein's (2008) 'Why model?', whose killer point is that a mathematical model at least gives a hypothesis that can be analyzed and falsified, in contrast to a purely verbal argument which may be too slippery to be tractable. Epstein also notes that a central purpose of simple mathematical models is to illuminate 'core dynamics' - that is, in dynamical systems, the ways that growth, decay, cyclicity and finally chaos appear. Whether in the FitzHugh-Nagumo model of an excitable system such as the human heart, the Kermack-McKendrick ('SIR') model of the epidemiology of a rapidly-developing

The original version of this chapter was revised: The name "Gregory D. Hess" has been corrected to "George D. Hess" in Reference list. The correction to this chapter is available at https://doi.org/10.1007/978-3-030-31589-4_12

This chapter builds on MacKay (2015). Permission granted by the Operational Research Society to reprint material from that article.

(C) The Author(s) 2020, corrected publication 2021

N. P. Gleditsch (ed.), Lewis Fry Richardson: His Intellectual Legacy

and Influence in the Social Sciences, Pioneers in Arts, Humanities, Science,

Engineering, Practice 27, https://doi.org/10.1007/978-3-030-31589-4_9 
infectious disease, the Lotka-Volterra model of predator-prey ecosystems or Alan Turing's model of pattern formation, an understanding of the essential dynamics is a necessary beginning and complement to more elaborate simulations. As so often, Lewis Fry Richardson himself put it well:

Strange to say, it is to the advantage of realism that mathematicians customarily replace the actual world by various idealized models. For they choose models that can be analyzed with ease; and thus they are free to think about the resemblances or misfits between the model and the actual world. If, with a solemn feeling of the importance of things as they really are, we were to admit the irregularities of the actual world into the statement of our problems, we should in consequence have to attend to enormous elaborations of mathematics in the process of solution, whereby our attention would for a long time be distracted away from the actual world (Richardson, 1960: 169).

But there is a stronger argument for the importance of simple models, namely that they necessarily capture the central truths hidden within more complex simulations, which, it must be understood, refine rather than supersede the simpler models. Approximations to complex models exist, describe their core dynamics, and can be classified. The classic example, post-dating Richardson, is Thom's 'catastrophe theory' from the 1960s, to which an excellent introduction is given by Poston \& Stewart (1978). This was heavily oversold at the time, but is nowadays undervalued for what it contains, a classification of all the ways that change can occur in dynamical systems. Indeed, it is no more than slightly simplistic to characterize catastrophe theory as a body of results about Taylor's theorem in higher dimensions - and Taylor's theorem, taught in school calculus classes, is no more than the writing down of the simplest approximation to any nonlinear behaviour: first a constant, then a linear variation, then a quadratic, and so on. In that light, Richardson's arms races are just the simplest, linear approximation to the dynamics of the tension between antagonism and fatigue. Of course, a dynamical system may not be the correct conception of such a situation, but to the extent to which it is so, Richardson's conclusions necessarily follow. As he himself said, 'All that can be proved by mathematics is that certain consequences follow from certain abstract hypotheses' (Richardson, 1960a: 145).

This chapter is based on two ideas: The first is a small conceit, an imagined meeting in the world of the intellect, of LFR's ideas with those of someone very different, the irascible and occasionally belligerent British engineer Frederick Lanchester, who wrote down the first models of attritional war, with their grim calculus of constant warfare and resulting annihilation (MacKay, 2015). The original motivation was the observation that rather similar core dynamics pervade various of the attempts in the literature to model attrition in insurgent warfare, in which the military forces are bound up with more nebulous, psychological variables. Many of these attempts can be encompassed in a combination of Richardson arms races with Lanchestrian attrition and its variants. The action is all in the scaling, in whether it is the attrition or the escalation which scales faster, and whether it does so asymmetrically in a consistent way.

The second is in the spirit of Richardson's generalization of his arms races from two to an arbitrary number of countries. His conclusion there was that 'the world 
will for most of the time be content with just enough stability' (Richardson, 1960: 183). I analyze general multilateral wars of attrition but present the situation here for just three antagonists. There is some history of modelling conflict among three actors, and the generalization of a pistol duel - the truel - throws up similar conclusions in most of its variants: when each actor's overriding objective is survival, it is often the weakest actor whose prospects are the best. Our conclusions, however, are in stark contrast: in Lanchestrian attrition, whether Square or Linear Law, with the objective of maximizing one's own numbers minus one's opponents' numbers at the end of the war, either one actor can beat the others put together, or mutual annihilation is the robust outcome.

\subsection{Richardson's Arms Race Model}

We begin by looking at the 'phase plane' of the Richardson arms race. For reasons which will become apparent we denote by $S$ and $R$ the variables $\mathrm{x}$ and y in his arms race model (cf. Smith, 2020, in this volume), here restricted to be positive. The phase plane then shows a field of arrows which denote the direction (but not the magnitude) of the flow of ( $S, R)$, and also shows the curves (for Richardson's model they are lines) on which the flow is either horizontal or vertical. These 'null clines' therefore intersect at points at which the flow is both horizontal and vertical: that is, where there is no flow, so that the intersections are the 'fixed points' of the system. Figure 9.1 shows the two qualitatively different possibilities. In Fig. 9.1a, on the left, fatigue outweighs antagonism. Whatever the starting point, the resulting flow reaches the stable fixed point, an equilibrium in which the two sides' forces are somewhat larger than would be the case in the absence of antagonism but are nonetheless stable. Figure $9.1 \mathrm{~b}$, on the right, illustrates what happens when antagonism increases: the null clines have passed through being parallel and now diverge, resulting, from all starting points, in a runaway arms race. I have heard it objected that there never has been an exponentially growing arms race of the kind implied, but of course the phase plot tells us nothing about the magnitude of the flow, and one can perfectly well nonlinearly rescale the time variable to alter the functional form of the rate of growth.

In (a) fatigue is predominating, resulting in a stable fixed point; in (b) antagonism is predominating, resulting in runaway arms growth. The null clines (of horizontal or vertical flow) are shown as thin red lines.

\subsection{A Lanchester-Richardson Model}

In Richardson's model the parameters which link the variables with their rates of change are psychological. A very different classic warfare model is that of Lanchester (1914), which describes attritional war. In Lanchester's 'aimed-fire' 
(a)

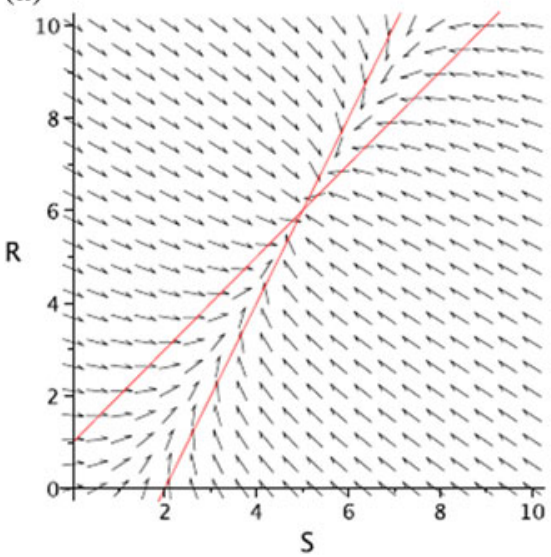

(b)

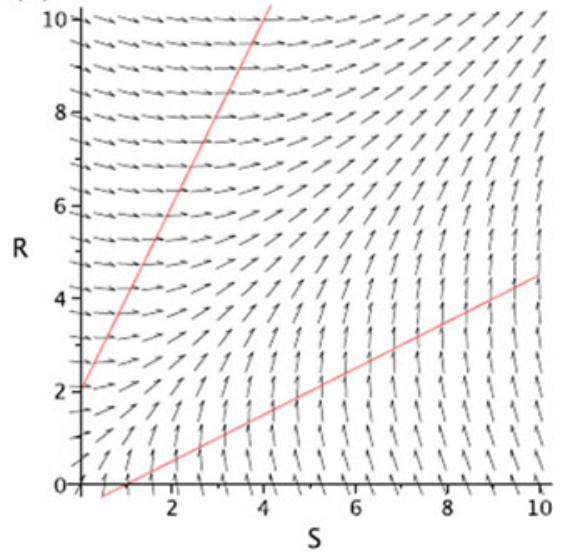

Fig. 9.1 The Richardson arms race

model, each force causes damage in proportion to its numbers, and the constant of proportionality is the rate at which each unit kills its enemies. There is little intrinsic interest in the phase plane, for there is no possibility of fixed points other than mutual annihilation $(S, R)=(0,0)$. The classic result, rather, results from the trajectories' being hyperbolae: the difference between the forces' kill-rates multiplied by the square of their numbers is constant, and thus determines the winner, in Lanchester's Square Law. Mathematically, of course, this is similar to Richardson's model, with altered parameters (merely), not altered (linear) dynamics: fatigue and constant grievance are absent, and antagonism has a negative effect on opponents.

Suppose that we combine the two models. At its simplest, the model is simply as in Fig. 9.1, but with antagonism reduced by kill-rate. The growth of the forces is now supplemented by the horror of continuous attrition to create either a stable equilibrium, or (if antagonism still outweighs all else) an arms race continuing during open war. Both situations are perhaps not so different from that of 1914-17: the fixed point in Richardson's model can just as well describe stasis in 'hot' war as in 'cold'. Nowhere in either phase plane do we see either side 'winning'.

Now suppose instead that we make the model asymmetric between $S$ and $R$, letting $S$ be the 'state' and $R$ the 'rebels' or 'revolution' in an insurgent war, and think about the conditions for a state win - that is, for the state to annihilate the rebels. Assume that the state uses its resources both to inflict high damage (in proportion to its own strength) on the rebels and to reduce the rate at which by its antagonism it causes the rebellion to grow, so that the former is greater than the latter. In contrast the state is able to reinforce itself in proportion to rebel numbers at a greater rate than it loses units to them. There are then two possible outcomes, whose controlling parameter is the (net negative) rate at which the state antagonises the rebellion minus the rate at which it kills the rebels. The result is, in Fig. 9.2a on the left, a low-antagonism regime which sees an inevitable state win, or, on the right in Fig. 9.2b, a high-antagonism regime which may see either (for sufficiently large 
(a)

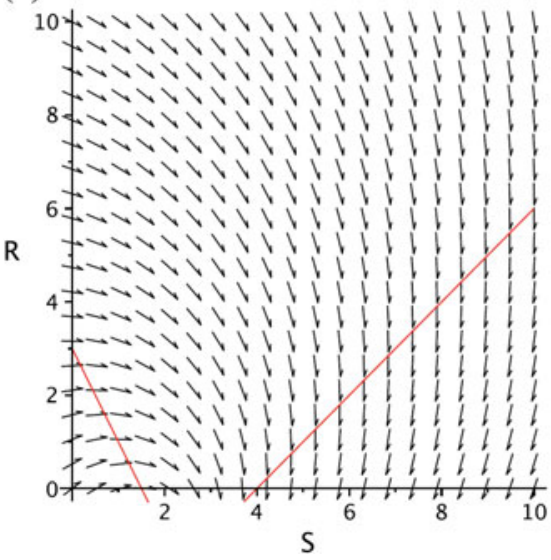

(b)

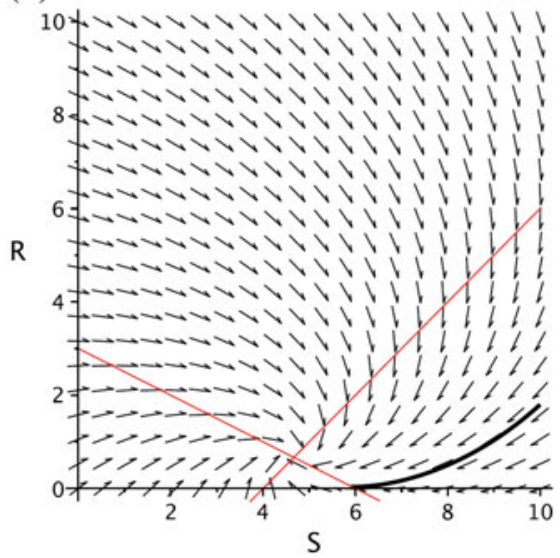

Fig. 9.2 A combined Lanchester-Richardson model. a Low antagonism, resulting in a state win, or $\mathbf{b}$ high antagonism, resulting either in a state win or - if the state is initially relatively weak - a fixed point (stalemate). The thin red lines are the null clines; the thick curve in (b) is the separatrix between stalemate and state win

initial $S$ and small initial $R$, to the right of the thick curve) a win for the state, or (otherwise) a fixed point, a stalemate.

This model captures some useful features. Set up in this way, it does not allow a rebel takeover. But it does distinguish an ongoing insurgency from a state win, with the latter requiring a reasonable set of conditions: effective attrition of the insurgency, low antagonism by the state, and a relatively strong initial state position. But (and this goes back to the issue of 'core dynamics'), it probably gets one crucial ingredient wrong: insurgencies almost certainly do not have the (symmetric) Lanchester square-law model as their attritional dynamics.

\subsection{A Deitchman-Richardson Model}

It is a standard feature of asymmetric variants of Lanchester models intended to describe insurgent or guerrilla warfare that (at its simplest) $R$ 's fire is 'aimed' whereas $S$ 's is 'unaimed', that the rebellion is able to target its fire more efficiently that the state (Deitchman, 1962). The upshot is an asymmetry of scaling, in which the state's action becomes relatively more effective when the insurgency is larger. The horizontal-flow null cline is then not a straight line but a curve, and the phase portraits are as shown in Fig. 9.3. Here both low antagonism (left, Fig. 9.3a) and high antagonism (right, Fig. 9.3b) result in a stalemate. There can be no unlimited escalation, for the asymmetric nonlinear scaling of the attrition of the rebellion by the state prevents it. 
(a)

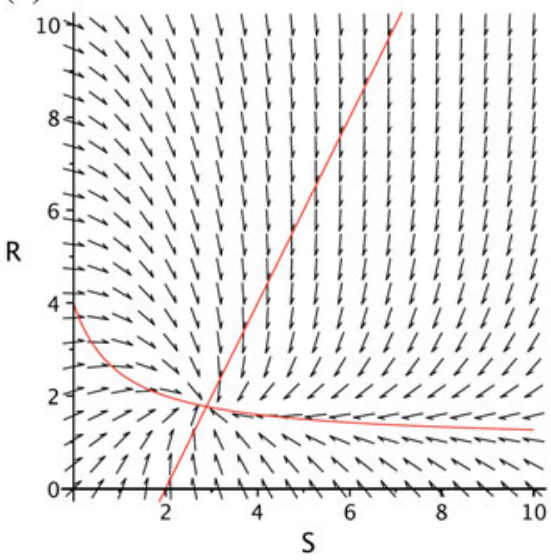

(b)

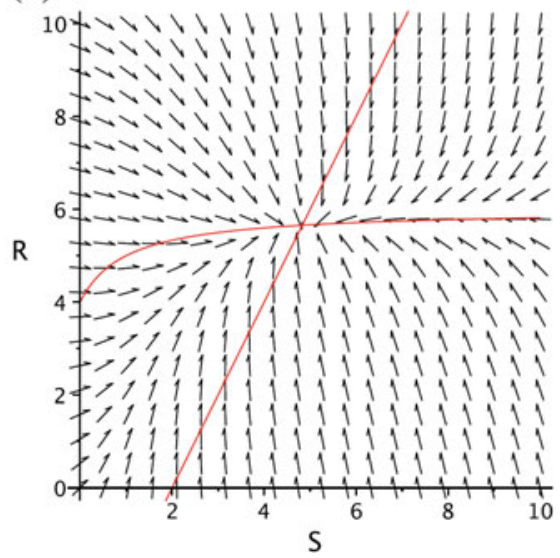

Fig. 9.3 A combined Deitchman-Richardson model has a stable fixed point for both a low antagonism and $\mathbf{b}$ high antagonism

In this Deitchman-Richardson model the only way for the state to win is for its ability to direct aimed fire - targeted, intelligent military action - at the rebellion to outweigh its antagonism. If this effect is sufficiently strong (Fig. 9.4a) the state always wins. If not (Fig. 9.4b) then there is, as in Fig. 9.2, a separatrix between the regime in which the state is sufficiently initially relatively strong to win and that in which a stalemate is reached.

These results are robust to more generalized scalings and subsume various results in the literature. Of course, a 2D dynamical system can do no more than illustrate some simplified dynamics and can anyway capture behaviour no more complex than growth, decay or a (perhaps cyclic) stale-mate. But the general conclusion is that simple Richardson-like linear antagonism, when combined with Lanchester-Deitchman theory in which the theoretical signature of insurgent warfare is that insurgent attrition scales faster than state attrition, typically results in stalemate.

This makes the crux of any empirical verification clear. Good time-series data on insurgent wars are hard to find: not only are the parties otherwise engaged, but the rebellion - both $R$ and its growth rate $\mathrm{d} R / \mathrm{dt}$ - remains largely hidden. But what one can do is to examine, for a set of insurgencies, the relationship between the level $S$ of state military force needed to win and the effectiveness rate $\mathrm{d} S / \mathrm{dt}$ of rebel military action. Goode (2009-10) did precisely this, analyzing 42 insurgencies. The theoretical expectation in Deitchman's model, deduced from its analogue of the Square Law, is that $S^{2} \sim R$ (where $\sim$ is to be read as 'scales like'), while $\dot{S} \sim R$ : combining these, $S \sim \sqrt{d S / d t}$ Goode found $S \sim(\mathrm{d} S / \mathrm{dt})^{0.45}$, a strikingly good match. 
(a)

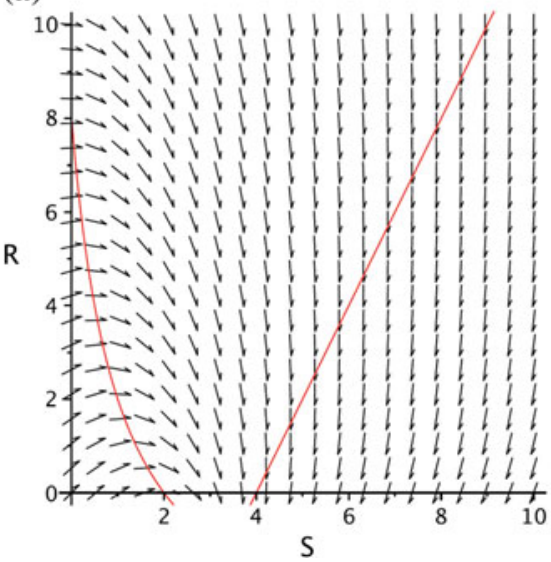

(b)

R

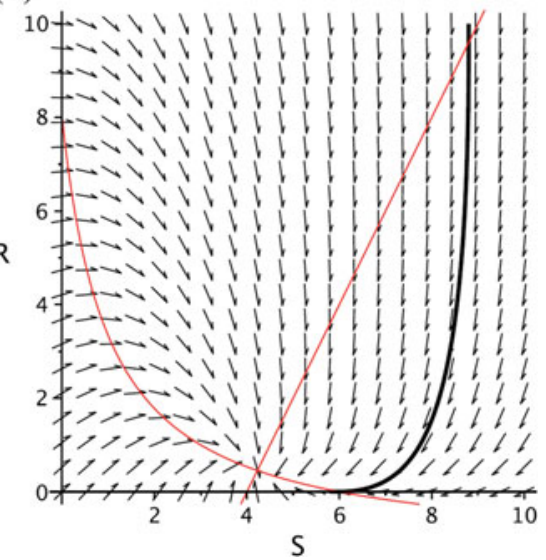

Fig. 9.4 Combined Deitchman-Richardson model with state targeted action greater than antagonism. In a when this effect is large, the state wins; in $\mathbf{b}$ when this effect is modest, the state wins if the initial rebellion is relatively small, else a stalemate is reached

\subsection{Models with Three Actors}

Richardson extended his model first to three and then to a larger general number of actors. In the case of three actors, suppose first that all individual actors have the same rate of fatigue, and the strengths of all pairwise antagonisms are equal. Pairwise, each interaction results in an arms race if and only if antagonism is greater than fatigue. Unfortunately, when we have three actors the situation is worse, for there is more antagonism going on - indeed it is, in a precise sense, doubled. An arms race occurs if and only if the pairwise antagonism is greater than half the individual rate of fatigue (Richardson, 1960: 154-155).

Now that we have three actors, however, we can introduce decision parameters and create differential games. We can also allow coalitions or alliances. A great deal of literature covers such possibilities, but a common thread runs through much of it: the apparent weakest actor is in many cases in the strongest position. For example, in a simple quantitative model of three forces, the largest force or coalition being the winner, it is typically the smallest force which has the greatest capacity to ensure it ends up on the winning side (Caplow, 1956: 490): 'the triadic situation often favors the weak over the strong'.

Battles among three actors, or 'truels', can be set up in many ways. Perhaps the very simplest is to give $A, B$ and $C$ each one shot and require them to act, in that order. What should $A$ do? If he shoots $B$, then $C$ will shoot him. If he shoots $C$, then $B$ will shoot him. Yet suppose he shoots in the air: then, by making himself powerless, he has become no threat to $B$, who shoots $C$, leaving $A$ and $B$ standing. Many variations - deterministic or probabilistic, with shooting in fixed or in random order - are possible, and it is often the worst shot or otherwise-weakest actor 
who has the greatest chance of success (Kilgour \& Brams, 1997). The underlying reason is that most actors value their own survival above all else, so that to be a threat is also to be a preferred target and thus to be in danger.

\subsection{The Lanchester Truel}

But here is a model in which this is not so: the 'Lanchester truel', a generalization of the Lanchester aimed-fire model in which each of three forces causes damage in proportion to numbers, but may divide its fire between its two opponents, and must decide how to do so for an optimal outcome. The model is thus

$$
\begin{aligned}
\mathrm{d} A / \mathrm{dt} & =-\mathrm{b}(1-\beta) B-\mathrm{c} \gamma C \\
\mathrm{~d} B / \mathrm{dt} & =-\mathrm{a} \alpha A-\mathrm{c}(1-\gamma) C \\
\mathrm{~d} C / \mathrm{dt} & =-\mathrm{a}(1-\alpha) A-\mathrm{b} \beta B
\end{aligned}
$$

where $A(\mathrm{t}), B(\mathrm{t}), C(\mathrm{t})$ are the force numbers and $a, b, c$ their individual kill-rates, all positive. The three forces begin with given numbers $A(0)=A_{0}, B(0)=B_{0}, C(0)=C_{0}$ units. Each unit of $A$ kills opponents (of either type) at rate $a, B$ does so at rate $b$, and $C$ at rate $c$. When one force is eliminated the other two fight a Lanchester aimed-fire duel, and the truel finishes when only one force remains. We'll call these final values, only one of which can be positive, $A_{\infty}, B_{\infty}$ and $C_{\infty}$.

All of the parameters described so far - the initial numbers $A_{0}, B_{0}, C_{0}$ and the kill-rates $a, b, c$ - are fixed for a given truel. But each force also has a decision parameter under its (and no one else's) control: for $A$ it is $\alpha$, for $B$ it is $\beta$, for $C$ it is $\gamma$. If $\alpha=1$ then $A$ targets only $B$; if $\alpha=0$ then $A$ targets only $C$; and the two are interpolated by $0 \leq \alpha \leq 1$. Each side can vary its parameter continuously throughout the fight if it wishes.

A set of coupled linear differential equations such as these is straightforward to solve, but the solution in itself is not very instructive (Kress et al., 2018a). The crucial first step is to decide on the objective. This is a war of attrition, but there is no equivalent, for general values of the parameters, of Lanchester's Square Law for this model - technically, this is because there is no quadratic quantity which remains the same throughout the battle, at least for general values of the parameters. Instead we take $A$ 's objective to be to maximize

$$
A_{\infty}-B_{\infty}-C_{\infty}
$$

and likewise for $B$ and $C$. That is, if $A$ can win then it wishes to do so with the largest possible remaining force, but if it cannot win then it wishes to minimize the sum of its opponents' remaining forces. It does so without discriminating between $B$ and $C$, but in fact this does not matter: the results apply to any objective function which is increasing in $A_{\infty}$ and decreasing in $B_{\infty}$ and $C_{\infty}$. Thus, the outcome in the 
end does not depend on the implied relative worth of one's own survival against one's opponents' destruction.

This outcome, in contrast to most truels and coalition models, is that either one side can beat the other two put together, or all three actors are locked in an attritional stalemate which leads to mutual annihilation. The reason is the valuing of the destruction of opponents by an actor which cannot emerge victorious. The proof is in two parts, and, while I shall not reprise the mathematical details, the essential content is easy to describe with the aid of Fig. 9.5. Here, for simplicity, I have projected $(A, B, C)$ onto the positive octant of the unit sphere, thereby concerning us only with the relative numbers of $A, B$ and $C$. In reality, the dynamics is, of course, of absolute numbers of $A, B$ and $C$ always declining.

Consider just one corner of this octant, where $A$ has very large numbers relative to $B$ and $C$, and $A$ dominates. That is to say, if $A$ chooses the correct fire distribution between $B$ and $C$, following the prescription of Lin \& MacKay (2014), then it can

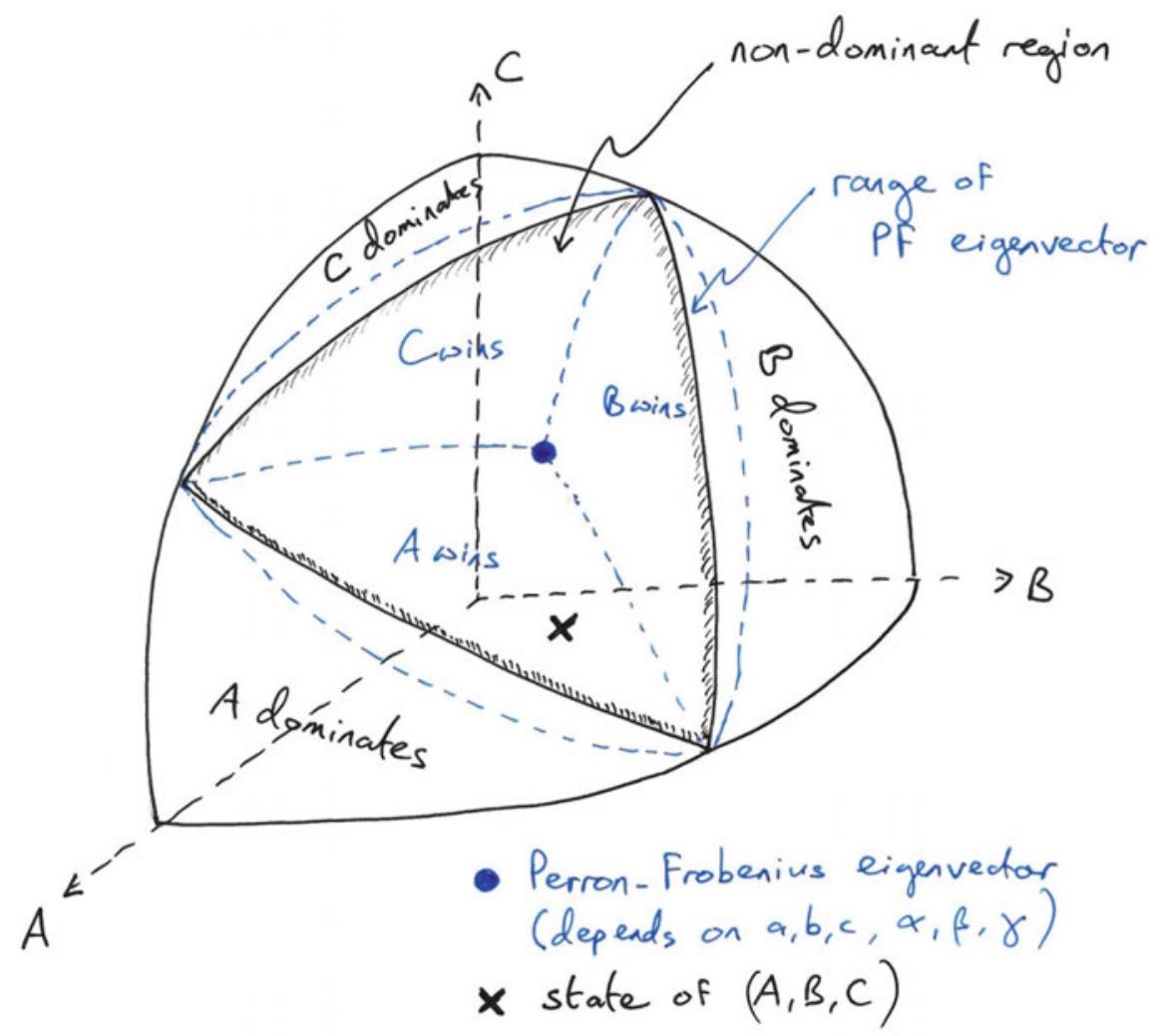

Fig. 9.5 The Lanchester truel. Force sizes A, B, C are projected onto the unit sphere. In the hachured triangle no one actor 'dominates' - that is, can beat the other two together. The game-theoretic outcome is that by altering their fire distributions the actors move the condition for collective mutual annihilation (dot) onto the present state (cross) 
guarantee to beat the other two forces even if they each fire only at $A$. Likewise for $B$ and $C$. These three regions bound (with the hachured lines) the central triangle, the non-dominant region, within which none of $A, B$ or $C$ can by their own choices guarantee to win.

Now consider the markings, which describe a lovely result in linear algebra. The dot is the Perron-Frobenius (or PF) eigenvector, the state from which the dynamics send $(A, B, C)$ directly in a straight line to the origin (thereby remaining static in the projection onto the unit sphere), to collective annihilation. Its position depends on the actors' policies $\alpha, \beta, \gamma$, and moves as these policies change. Within what bounds can it move? These are shown as the dashed lines, and it is an elegant result that the range of the dot, the PF eigenvector, bounded by the dashed lines, strictly includes the non-dominant region, with equality when $a=b=c$.

The rest of the result is a straightforward differential game. The dot is a Nash equilibrium, a position from which, within the non-dominating region, none of $A, B$ or $C$ can do better (according to their objectives) by departure; and the combined action of $A, B$ and $C$ will always push the dot onto whatever is the current state (marked as a cross on the figure). The combined effect of the actors optimizing their policies is for the dot (the state from which the outcome is mutual annihilation) to chase the cross (the current state) wherever it goes. This gives the result a great deal of robustness: the present state (cross) may move, but the annihilating state (dot) will chase it. Departures of the cross from the dot could be caused by small reinforcements, changes in kill rates, missteps in policy; whatever. But as long as the cross does not move into a dominating region, and as long as the actors can change their policies more rapidly than attrition changes the state of the forces, the dot will catch up with the cross and remain there. In fact, the result is much more general even than this: it applies to a general number of actors, to kill-rates which depend on opponent as well as on attacker, and to Lanchester's 'ancient' (linear law) model of warfare as well as to his aimed fire (Square Law) model (Kress, Lin \& MacKay, 2018b).

Where does this leave the original truel insight, that the weakest is paradoxically strong? Can this be replicated in the Lanchester truel? In fact, it can, but one needs to choose a different objective function. In the Lanchester one-against-many problem, the optimal policy can be fixed by thinking about quadratic quantities, but it is identical with the policy produced by trying to maximize the rate of reduction of one's own casualty rate. Here this would amount to $A$ trying to maximize $\mathrm{d}^{2} A$ / $\mathrm{dt}^{2}$, and likewise for $B$ and $C$. Suppose one takes this to be the objective. One can then impose a differential game in which each side alters its policy so as to maximize its own casualty-rate reduction. Let $a>b>c$, so that $A$ is the most individually-effective force, followed by $B$, then $C$. It turns out that if $A$ 's and $B$ 's numbers are approximately balanced, $C$ can judiciously divide its fire between $A$ and $B$ and emerge the winner. Why do $A$ and $B$ not also fire at $C$ ? Essentially because $A$ is the bigger threat to $B$, and vice versa: and derogation by $A$ and $B$ from concentrating on each other, by firing at $C$, will only make their casualties worse. Within the cube $0 \leq \alpha, \beta, \gamma \leq 1$ of fire policies, this is the outcome with the largest basin of attraction; there is a smaller line-segment for which the same is true 
of an $A$ - $C$ duel with $B$ looking on; and, only for $b+c>a$, a small line-segment of stable fixed points of $B-C$ duel with $A$ looking on.

The action, then, is all in the objective function: the overall conclusion is that, in multilateral war, if actors value their opponents' destruction as well as their own survival, then truel-like results no longer hold: rather, if no one actor can beat all the others put together, then all of them are headed for mutual destruction. If, instead, actors value only their own hurt, then something like classic truel results may follow. One can also show that, if your opponents value your destruction but you do not value theirs, then you must also inflict hurt on them (even if your objective is only to avoid your own hurt); it does no good with such opponents to withhold any of your own fire.

\subsection{Concluding Thoughts}

A fine review of Richardson's arms race models, including the multi-actor case and connecting it to the modern theory of dynamical systems and properties of the associated matrices, is given by Hess (1995). The recent literature on the modelling of insurgent war, much of which falls under the scope of generalized Lanchester-Richardson models (MacKay, 2015), still has much to learn from Richardson's work. Indeed, there are many more connections to be made in work on multilateral conflict, not only between arms races and attritional war, but also with the literature on stability in complex ecosystems which has grown from the pioneering work of May (1973), and even, in ongoing work which brings attention full-circle back to pressing questions of human social structures, with the stability of banking systems (Haldane \& May, 2011; Bardoscia et al., 2017).

\section{References}

Bardoscia, Marco; Stefano Battiston, Fabio Caccioli \& Guido Caldarelli (2017) Pathways towards instability in financial networks. Nature Communications 8: 14416.

Caplow, Theodore (1956) A theory of coalitions in the triad. American Sociological Review 21(4): 489-493.

Deitchman, Seymour (1962) A Lanchester model of guerrilla warfare. Operations Research 10(6): 818-827.

Epstein, Joshua (2008) Why model? Journal of Artificial Societies and Social Simulation 11(4): 12.

Goode, Steven M (2009-10) A historical basis for force requirements in counterinsurgency. Parameters (Winter): 45-57.

Haldane, Andrew \& Robert May (2011) Systemic risk in banking ecosystems. Nature 469(7330): 351.

Hess, George D (1995) An introduction to Lewis Fry Richardson and his mathematical theory of war and peace. Conflict Management and Peace Science 14(1): 77-113.

Kilgour, D Marc \& Steven J Brams (1997) The truel. Mathematics Magazine 70(5): 315-326.

Kress, Moshe; Jonathan Caulkins, Gustav Feichtinger, Dieter Grass \& Andrea Seidl (2018a) Lanchester model for three-way combat. European Journal of Operational Research 264(1): 46-54. 
Kress, Moshe; Kyle Lin \& Niall MacKay (2018b) The attrition dynamics of multilateral war. Operations Research 66(4): 950-956.

Lanchester, Frederick (1914) Aircraft in Warfare: The Dawn of the Fourth Arm. London: Constable. [Based on 1913-14 articles in Engineering 98: 422-423 and 452-453. The chapter which deals with the mathematical models is reprinted in James Newman (ed.) (1956) The World of Mathematics 4: 2138-2157. New York: Simon and Schuster, reprinted by New York: Dover, 2000.]

Lin, Kyle \& Niall MacKay (2014) The optimal policy for the one-against-many heterogeneous Lanchester model. Operations Research Letters 42: 473-477.

MacKay, Niall (2015) When Lanchester met Richardson, the outcome was stalemate: A parable for mathematical models of insurgency. Journal of the Operational Research Society 66: 191201. [Reprinted as Ch. 6 of RA Forder (ed.) (2015) Operational Research, Defence and Security. Operational Research Essentials. London: Palgrave Macmillan, in association with the Operational Research Society: 124-147.]

May, Robert (1973) Stability and Complexity in Model Ecosystems. Princeton, NJ: Princeton University Press.

Poston, Tim \& Ian Stewart (1978) Catastrophe Theory and Its Applications. London: Pitman.

Richardson, Lewis F (1960) Arms and Insecurity: A Mathematical Study of the Causes and Origins of War. Pittsburgh, PA: Boxwood.

Smith, Ron P (2020) The influence of the Richardson arms race model. Ch. 3 in this volume.

Niall MacKay, b.1967, B.A. in Mathematics (Cambridge, 1988), Ph.D. in Theoretical Physics (Durham, 1992); Professor and Head of Department of Mathematics, University of York; publications in mathematical physics, operations research and history, niall.mackay@york.ac.uk.

Open Access This chapter is licensed under the terms of the Creative Commons Attribution 4.0 International License (http://creativecommons.org/licenses/by/4.0/), which permits use, sharing, adaptation, distribution and reproduction in any medium or format, as long as you give appropriate credit to the original author(s) and the source, provide a link to the Creative Commons license and indicate if changes were made.

The images or other third party material in this chapter are included in the chapter's Creative Commons license, unless indicated otherwise in a credit line to the material. If material is not included in the chapter's Creative Commons license and your intended use is not permitted by statutory regulation or exceeds the permitted use, you will need to obtain permission directly from the copyright holder. 